\title{
Helminth infection ameliorates arthritis
}

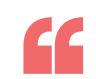

\section{Nippostrongylus brasiliensis} infection ... inhibited both arthritis and bone loss
A new study published in Nature Communications describes how arthritis in mice is attenuated following helminth infection. Nippostrongylus brasiliensis infection led to a $\mathrm{T}$ helper type $2\left(\mathrm{~T}_{\mathrm{H}} 2\right)$ response and eosinophil accumulation in the joints, and inhibited both arthritis and bone loss in two mouse models of arthritis (a serum-transfer model and a human-TNF transgenic model). "Our most important finding is that $\mathrm{T}_{\mathrm{H}} 2$ cells and eosinophils both contribute to resolution of arthritis," note Aline Bozec and Georg Schett, corresponding authors of the study.

The importance of eosinophils in the helminth-infection-induced resolution of arthritis (which the researchers' report states is probably mediated by increased ingress of anti-inflammatory macrophages into arthritic joints) came as a surprise.

"They actually show that the eosinophils per se are the agents delivering the beneficial effects," highlights Yehuda Shoenfeld, who was not involved in the study.

The study shows that the protective effect of helminth infection is dependent on activation of the STAT6 (signal transducer and activator of transcription 6) pathway in haematopoietic cells, by 'alternative' cytokines, namely IL-4, IL-13 and IL-5. This activation of the STAT6 pathway shifts macrophage polarization towards an anti-inflammatory phenotype, and these cells contribute to the modulation of arthritis.

The researchers also identified $\mathrm{T}_{\mathrm{H}} 2$ cells and eosinophils in the synovial membranes of patients with rheumatoid arthritis, suggesting that these pathways are also relevant in human arthritis.

Schett and Bozec highlight that the next step is to determine whether it is possible to define an immune signature for resolution in patients with rheumatoid arthritis. "What is particularly intriguing is that a specific immune mechanism which is seminally involved in the pathogenesis of asthma and other immunemediated diseases is stopping arthritis," comments Schett - and Shoenfeld concurs: "This study raises several crucial questions: for instance, how is it that we have autoimmune diseases associated typically with eosinophilia (i.e. Churg-Strauss vasculitis)? Why are autoimmune diseases associated with an increased incidence of allergic disease (and eosinophilia)?"

To explore these and other questions, further studies are warranted, Shoenfeld notes, including analysis of different types of eosinophils, studying the effects of secreted material from eosinophils, and performing additional studies of eosinophil transfer. "Such studies might explain why other compounds secreted from helminths benefit autoimmune disease without association of eosinophils," he comments. Shoenfeld's group is investigating the tolerogenic effects of helminthderived compounds. "We believe for instance that a helminth-derived compound containing phosphorylcholine most probably induces eosinophilia," asserts Shoenfeld.

Caroline Barranco

ORIGINAL ARTICLE Chen, Z. et al. Th2 and eosinophil responses suppress inflammatory arthritis. Nat. Commun. http://dx.doi.org/10.1038/ ncomms11596 (2016)

FURTHER READING Bashi, T. et al. Novel

therapeutic compound tuftsin-phosphorylcholine attenuates collagen-induced arthritis. Clin. Exp. Immunol. 184, 19-28 (2016)

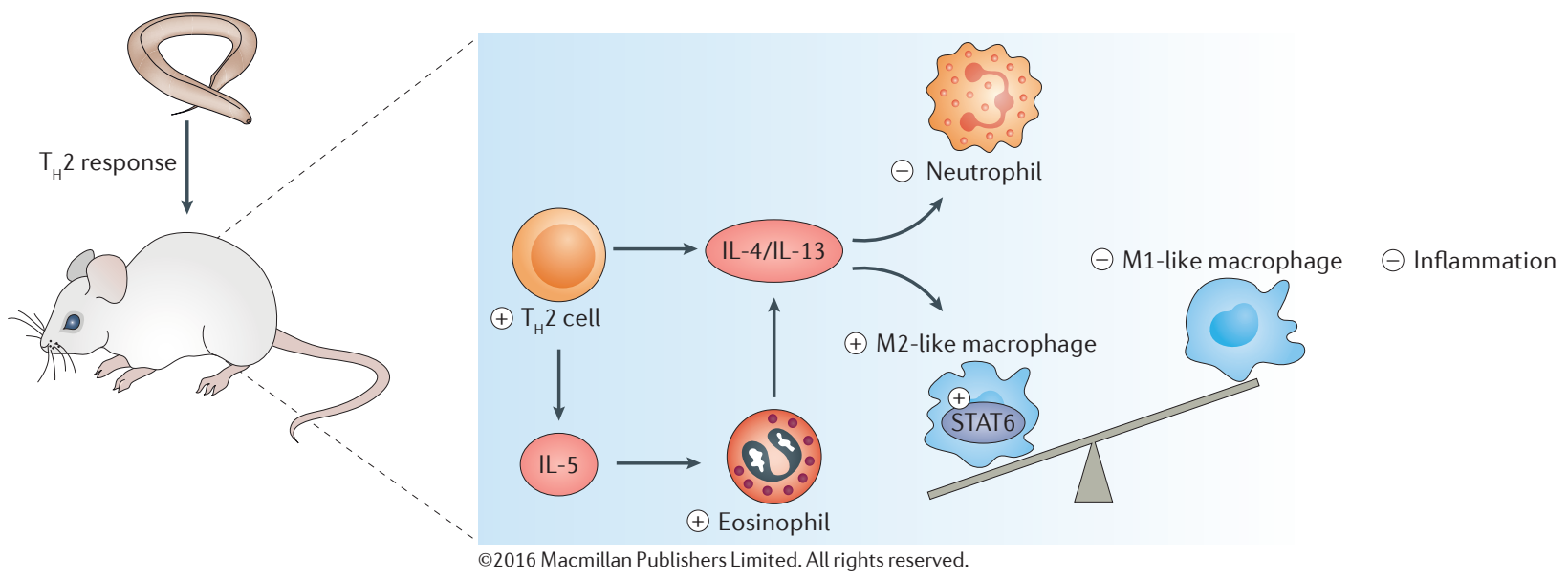

